\title{
Influence of protein kinase RIPK4 expression on the apoptosis and proliferation of chondrocytes in osteoarthritis
}

\author{
LIXUE ZOU, JUN LIU and HOUGEN LU \\ Department of Orthopedic Surgery, Jingzhou Central Hospital, Jingzhou, Hubei 434020, P.R. China
}

Received July 21, 2017; Accepted November 21, 2017

DOI: $10.3892 / \mathrm{mmr} .2017 .8209$

\begin{abstract}
The present study aimed to investigate the expression of receptor-interacting protein kinase 4 (RIPK4) and its effect on the apoptosis and proliferation of chondrocytes in osteoarthritis (OA). A total of 28 OA cartilage tissues and 20 normal cartilage tissues were collected to detect the expression of RIPK4 by using reverse transcription-quantitative polymerase chain reaction and western blot analysis. Chondrocytes were isolated from OA cartilage tissues and divided into OA, NC, si-RIPK4, Wnt3a, and si-RIPK4+Wnt3a groups, and those isolated from normal cartilage tissues were considered the Normal group. Chondrocytes proliferation was detected by MTT assay, cell apoptosis was indicated using flow cytometry and $\mathrm{Wnt} / \beta$-catenin signaling pathway related-proteins were investigated using western blot analysis. RIPK4 mRNA and protein expression levels in OA cartilage tissues and OA chondrocytes were increased compared with normal controls (all $\mathrm{P}<0.05$ ). Additionally, OA chondrocytes showed reduced cell proliferation, increased cell apoptosis and upregulated expression levels of Wnt/ $\beta$-catenin signaling pathway related-proteins (all $\mathrm{P}<0.05)$. Once transfected with si-RIPK4, the proliferation ability of chondrocytes was enhanced, but apoptosis was notably decreased. Furthermore, the expression levels of Wnt/ $\beta$-catenin signaling pathway related-proteins were significantly downregulated (all $\mathrm{P}<0.05)$. Results indicated that Wnt3a reversed the effect of si-RIPK4 on chondrocyte proliferation and apoptosis (all $\mathrm{P}<0.05)$. Thus, silencing RIPK4 promoted the proliferation and inhibited the apoptosis of chondrocytes. In addition, silencing RIPK4 blocked the $\mathrm{Wnt} / \beta$-catenin signaling pathway, thus contributing to alleviating the OA pathogenesis.
\end{abstract}

Correspondence to: Mr. Lixue Zou, Department of Orthopedic Surgery, Jingzhou Central Hospital, 60 Jingzhong Road, Jingzhou, Hubei 434020, P.R. China

E-mail: zoulixue66@hotmail.com

Key words: RIPK4, osteoarthritis, chondrocyte, proliferation, apoptosis, Wnt/ $\beta$-catenin

\section{Introduction}

Osteoarthritis (OA) is a chronic joint disease, characterized by degeneration of articular cartilage and secondary bone hyperplasia, ultimately resulting in stiffness, swelling, pain, and functional incapacitation of joints (1). As one of the most common types of arthritis, OA usually occurs in weight-bearing joints of the lower extremities, such as the hip joints and knee joints (2). As recorded, the risk of disability caused by OA is approximately $40 \%$ in males and $47 \%$ in females, which would be higher in obese people (3). With the rapid aging of population, more and more middle-aged and elderly people are bothered by OA, seriously affecting the quality of life of patients $(4,5)$. Despite the etiology of OA is very complicated and has not been clearly clarified, a variety of factors can lead to the development of OA, like joint trauma, abnormal mechanical burden, poor nutrition and genetic predisposition (6,7). At present, most of the medicine used to treat OA can temporarily relieve the pain of joints, but cannot improve the destruction of inflammatory joints (8). Therefore, it is important to find the molecular targets for the development and progression of $\mathrm{OA}$, and to create new therapeutic strategies for improving the treatment and prognosis of $\mathrm{OA}$ patients.

Receptor-interacting protein kinase 4 (RIPK4) is a family member of the receptor-interacting kinase proteins, which have served as crucial sensors of intracellular and extracellular stresses (9). Located on chromosome 21q22.3 and consisted of 8 exons and 7 introns, RIPK4 gene can encode $82 \mathrm{kd}$ proteins (10). RIPK4, as the important apoptosis modulator, contains an N-terminal RIP-like kinase domain and a $\mathrm{C}$-terminal region, which is characterized by the presence of 11 ankyrin repeats $(11,12)$. To our knowledge, RIPK4 is widely expressed in embryonic and mature tissues, such as myocardium, liver, skeletal muscle and lung (13), as well as involved in a variety of signaling pathways to play a vital part in regulating cells apoptosis, proliferation, differentiation and inflammatory response (14). So far, mounting evidence have shown that RIPK4 is up-regulated in various tumor tissues, which can promote the occurrence and progression of many malignant tumors, such as ovarian cancer (15) and cervical cancer (16). Besides, RIPK4 is also required for the formation of keratinocytes, the differentiation of epithelial cells, the skin inflammation, the healing of wound surface, as well as the tumor differentiation $(11,17,18)$. Although the occurrence 
of OA is closely related to chondrocyte apoptosis as suggested by the previous study (19), there is no article reported whether RIPK4 plays a regulatory role in the chondrocytes of OA until now.

Therefore, this study is to investigate the expression of RIPK4 and its effect on the apoptosis and proliferation of chondrocytes during the $\mathrm{OA}$, whereby providing a reliable experimental basis for the clinical use of gene therapy to treat OA.

\section{Materials and methods}

Ethics statement. This study was in accordance with the Helsinki Declaration (20), all patients were informed the purpose of the study and signed an informed consent before participation. The experiments in the study were approved by the Ethics Committee of Jingzhou Central Hospital (Jingzhou, China).

Study subjects. From December 2015 to December 2016, we collected 28 specimens of degenerated cartilage tissues from 10 male and 18 female patients (mean age: 56.8 \pm 8.7 years) who underwent arthroscopic debridement or total knee arthroplasty due to knee OA. Patients were diagnosed according to the OA diagnostic criteria issued by the American College Rheumatology (ACR) in 1995 (21). At the same time, 20 specimens of normal joint tissues were obtained as healthy controls from 8 males and 12 females (mean age: 57.4 10.1 years) who received amputation due to severe trauma to the lower extremities. There was no significant difference in the sex ratio and age between the patients with OA and the normal controls (all $\mathrm{P}>0.05)$.

Chondrocyte isolation and culture. Articular cartilage was cut into pieces under sterile condition before adding $0.2 \%$ type-II collagenase (Sigma, USA) of 10 times volume for $3 \sim 4 \mathrm{~h}$ of stirring and digestion at $37^{\circ} \mathrm{C}$. Next, 5,200 mesh sieve was used to filter the cell suspension, which was centrifuged for $10 \mathrm{~min}$ at the rate of 1,200 $\mathrm{xg}$ to remove the supernatant. Then, cells were washed for 2 times with $10 \%$ serum culture medium, and the cells obtained were cultured in Dulbecco's modified Eagle's medium (DMEM) containing 10\% fetal bovine serum and placed in $37^{\circ} \mathrm{C}, 5 \% \mathrm{CO}_{2}$ incubator with saturated humidity.

Cell transfection and grouping. Cells under good state were spread on 6-well plate. When the cell density reaches $80 \%$, transfection experiment was conducted with transfection mixture prepared according to the instructions on Lipofectamine $^{\circledR} 2000$ (Invitrogen; Thermo Fisher Scientific, Inc., Waltham, MA, USA) kit. The medium solution was discarded, prepared transfection mixture was added into the plate, and cells were cultured at $37^{\circ} \mathrm{C}$. Cells were classified into six groups: i) Normal group (normal articular chondrocytes); ii) OA group (OA chondrocytes); iii) NC group (OA chondrocytes transfected with negative control); iv) si-RIPK4 group (OA chondrocytes transfected with si-RIPK4); v) Wnt3a group [OA chondrocytes treated with a Wnt signaling agonist Wnt-3a (100 ng/ml)]; and vi) si-RIPK4+Wnt3a group (OA chondrocytes treated with si-RIPK4 and the Wnt signaling agonist Wnt-3a). The siRNA target sequence used to effectively inhibit the expression of RIPK4 and negative control
(NC) sequence were purchased from Shanghai Genechem Co., Ltd. (Shanghai, China).

Quantitative PCR detects the mRNA expression of RIPK4. The total RNA extracted from joint tissues or cells by Trizol reagent (Invitrogen; Thermo Fisher Scientific, Inc.) was determined for RNA concentration and purity. Sample RNA was reversed to cDNA in accordance with instructions on the reverse transcription kit (cat. no. DRR047S; Takara Biotechnology Co., Ltd., Dalian, China) and the total system volume was $10 \mu 1$. Then $65 \mu \mathrm{l}$ of DEPC-treated water was added into the cDNA for dilution and fully mixing. qPCR reaction system contains $5 \mu \mathrm{l}$ SsoFast EvaGreen Supermix (cat. no. 1708882; Bio-Rad Laboratories, Inc., Hercules, CA, USA), $0.5 \mu$ forward primer $(10 \mu \mathrm{M}), 0.5 \mu \mathrm{l}$ reverse primer $(10 \mu \mathrm{M})$, and $4 \mu \mathrm{l} \mathrm{cDNA}$. PCR amplification conditions included 40 cycles of pre-degeneration at $95^{\circ} \mathrm{C}$ for $5 \mathrm{~min}$, degeneration at $94^{\circ} \mathrm{C}$ for $60 \mathrm{sec}$, annealing at $55^{\circ} \mathrm{C}$ for $60 \mathrm{sec}$, and extending at $72^{\circ} \mathrm{C}$ for $120 \mathrm{sec}$. Primers were synthesized by Beijing Genomics Institute (BGI, Beijing, China). The primers of RIPK4 were 5'-GGATGCCCACTA CCACGTCA-3' and 5'-TGCCAAACAGGCCATCCA-3'. GAPDH was the internal reference gene and its primers were 5'-CTACCCACGGCAAGTTCAAT-3' and 5'-GGATGCAGG GATGATGTTCT-3'. Each gene of every sample would have three repeats. The reliability of PCR results was evaluated by melting curve. $\mathrm{Cq}$ value (inflection point of amplification curve), $\Delta \mathrm{Cq}\left(=\mathrm{Cq}_{\text {target gene }}-\mathrm{Cq}_{\text {internal reference gene }}\right), \Delta \Delta \mathrm{Cq}\left(=\Delta \mathrm{Cq}_{\text {case }}\right.$ group $\left.-\Delta C \mathrm{q}_{\text {control group }}\right)$, and $2^{-\Delta \Delta \mathrm{Cq}}$ was used to calculate the relative expression of target genes (22). The experiment was repeated for three times.

The protein expression detected by western blotting. The total protein extracted from joint tissues or cells was detected for the protein concentration by BCA kit (Beyotime Biotechnology Co., Ltd., Shanghai, China). Then, 5X SDS loading buffer was added for $5 \mathrm{~min}$ of denaturation at $95^{\circ} \mathrm{C}$. Next, SDS-PAGE electrophoresis was conducted before transferring membrane and adding $5 \%$ skim milk for closure overnight at $4^{\circ} \mathrm{C}$. Then, after membrane washing with Tris-Buffered Saline Tween-20 (TBST), the following primary antibodies (all in 1:1,000 dilution) were added respectively, including RIPK4 (ab84365; Abcam, Cambridge, UK), GSK-3 $\beta$ (ab68476; Abcam), $\beta$-catenin (ab6302; Abcam), Wnt3a (ab28472; Abcam), and $\beta$-actin (ab8226; Abcam). After incubation overnight at $4^{\circ} \mathrm{C}$, membranes were washed with TBST and secondary antibody horseradish peroxidase (HRP) was added to incubate for $1 \mathrm{~h}$ at $37^{\circ} \mathrm{C}$. At last, the membrane was washed with TBST and horseradish peroxidase ECL was added for development, scanning and recording. Image $\mathrm{J}$ software (NIH Image; National Institutes of Health, Bethesda, MD, USA) was used to analyze the gray value of target bands. The experiment was repeated three times independently.

Cell proliferation detected by MTT assay. The chondrocytes being transfected were diluted to a certain concentration and spread on 96 -well plates with $5 \times 10^{3}$ cells in each well. The plates were placed in $37^{\circ} \mathrm{C}, \mathrm{CO}_{2}$ incubator for $24,48,72$, and 96 h, respectively. Next, $20 \mu \mathrm{l}$ MTT reagent (Promega Corporation, Madison, WI, USA) was added into each well for another 1-4 $\mathrm{h}$ of incubation. Then, the absorbance value 

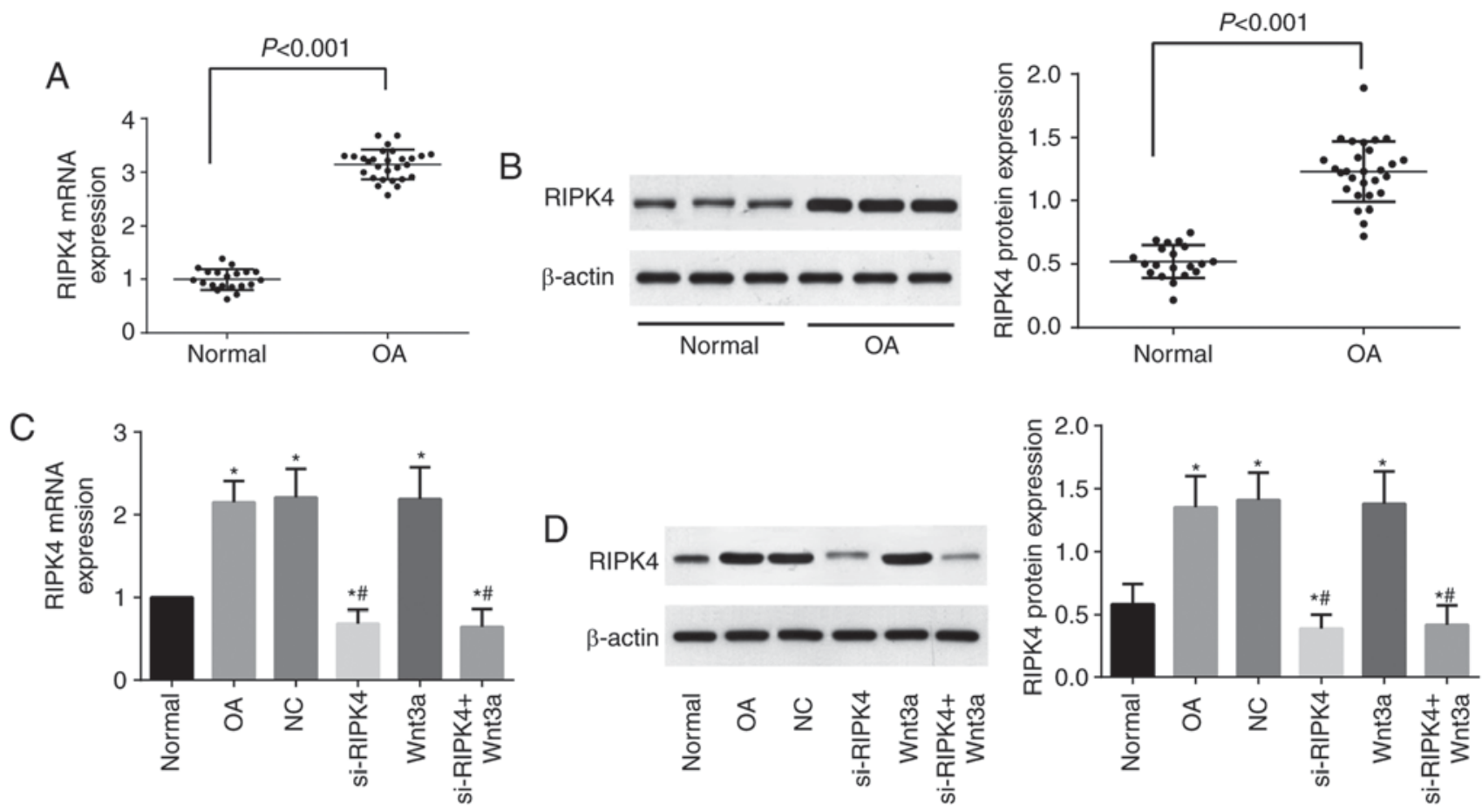

Figure 1. Expression of RIPK4 in cartilage tissues of OA patients and healthy controls and chondrocytes of each transfected group. (A) RIPK4 mRNA expression in cartilage tissues of OA patients and healthy controls was detected by qPCR; (B) RIPK4 protein expression in in cartilage tissues of OA patients and healthy controls was detected by western blotting; (C) The mRNA expression of RIPK4 in chondrocytes of each transfected group was detected by qPCR; (D) The protein expression of RIPK4 in chondrocytes of each transfected group was detected by Western blot; ${ }^{*} \mathrm{P}<0.05$ compared with Normal group; ${ }^{*} \mathrm{P}<0.05$ compared with the OA group. RIPK4, receptor-interacting protein kinase 4; OA, osteoarthritis.

was determined by the multifunctional microplate under the wavelength of $490 \mathrm{~nm}$.

Cell apoptosis rate detected by flow cytometry. Cells transfected for $24 \mathrm{~h}$ were digested by trypsin, centrifuged, and collected. The cells obtained were washed with cold PBS and made into $1 \times 10^{6}$ cells $/ \mathrm{ml}$ single cell suspension with $500 \mu \mathrm{l}$ binding buffer solution (calcium containing PBS). At room temperature, $100 \mathrm{ul}$ of cell suspension was added into the tube, followed by $5 \mu 1$ Annexin V-FITC (BD Biosciences, Franklin Lakes, NJ, USA) and $5 \mu 1$ propidium iodide (PI) (BD Biosciences). After $30 \mathrm{~min}$ of incubation at $4^{\circ} \mathrm{C}, 400 \mu \mathrm{l}$ binding buffer was added immediately before detection by the flow cytometry (BD Biosciences), $10^{4}$ cells each time. The data was analyzed using Cell Quest (BD Biosciences) to calculate the rate of cell apoptosis.

Statistical analysis. The data were analyzed with the statistical software SPSS 21.0 (SPSS, Inc, Chicago, IL, USA). Measurement data were presented by mean \pm standard deviation. Difference between two groups of measurement data that obey normal distribution was analyzed by Student's t-test, while comparison among multiple groups was conducted by one-way ANOVA. $\mathrm{P}<0.05$ was considered to indicate a statistically significant difference.

\section{Results}

Expression of RIPK4 in cartilage tissues and chondrocytes. As shown in Fig. 1, the mRNA and protein expression of RIPK4 in OA cartilage tissues was significantly upregulated

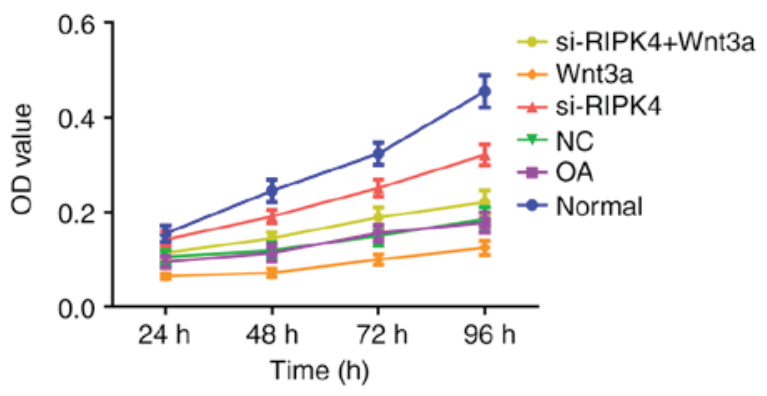

Figure 2. Comparison of the chondrocytes proliferation in each transfected group was detected by MTT. As compared to Normal group, the proliferation ability of chondrocytes in OA group and NC group was obviously reduced. Besides, the proliferation ability of chondrocytes was appreciably enhanced in si-RIPK4 group but obviously decreased in Wnt3a group when compared with the OA group, and Wnt3a could reverse the effect of si-RIPK4 on chondrocyte proliferation. RIPK4, receptor-interacting protein kinase 4; OA, osteoarthritis; NC, negative control.

as compared with normal cartilage tissues $(\mathrm{P}<0.05)$. In addition, we also examined the expression of RIPK 4 in chondrocytes after transfection, and by comparison with the Normal group, the expression of RIPK4 was elevated dramatically in the OA group, $\mathrm{NC}$ group, and Wnt3a group (all $\mathrm{P}<0.05$ ), but apparently reduced in the si-RIPK4 group and si-RIPK4+Wnt3a group $(\mathrm{P}<0.05)$. Moreover, RIPK4 expression in the si-RIPK4 and si-RIPK4+Wnt3a groups was remarkably lower than that in the OA group and the NC group (all $\mathrm{P}<0.05$ ), although there was no significant alterations in Wnt3a group $(\mathrm{P}>0.05)$. Chondrocytes proliferation in each transfected group. 

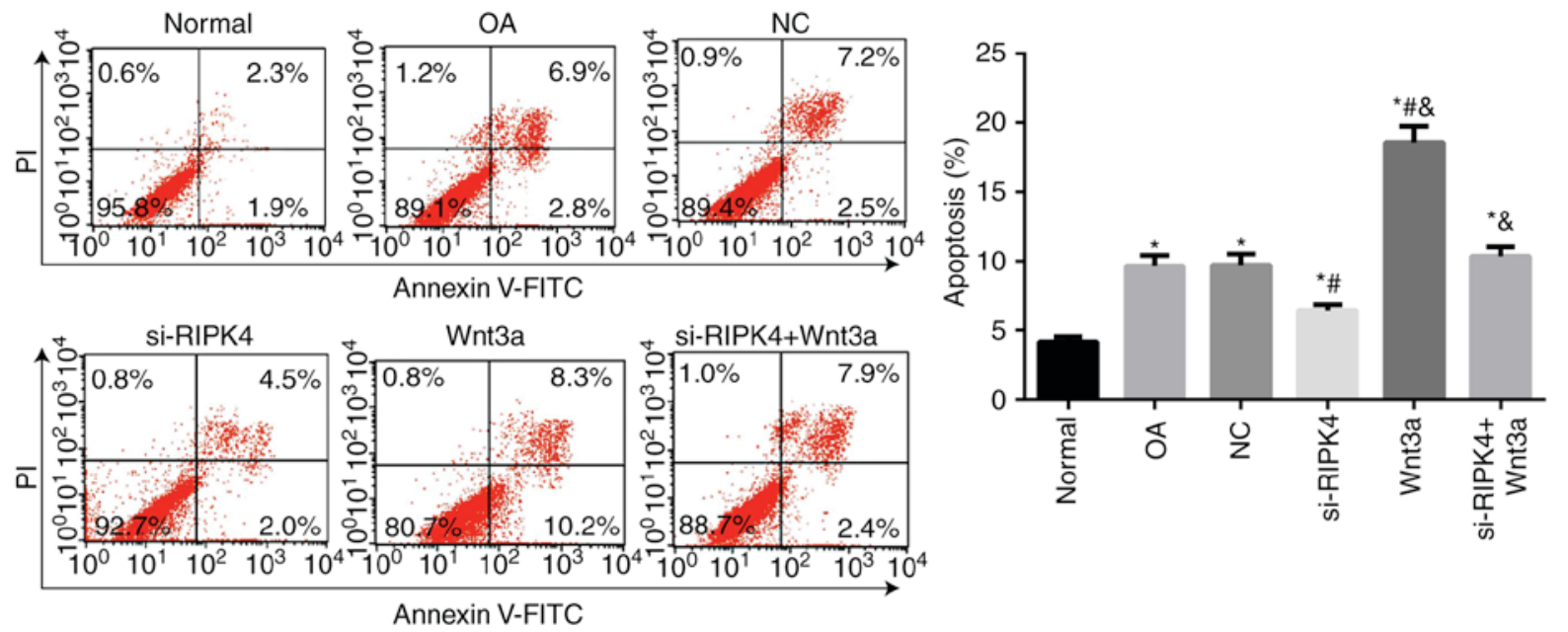

Figure 3. Chondrocytes apoptosis in each transfected group was detected by flow cytometry. si-RIPK4 effectively delayed the apoptosis of chondrocytes, but Wnt3a promoted the chondrocyte apoptosis, and Wnt3a reversed the effect of si-RIPK4 on chondrocyte apoptosis. "P<0.05 compared with normal group; ${ }^{\text {"P }} \mathrm{P}<0.05$ compared with the OA group; ${ }^{\circ} \mathrm{P}<0.05$ compared with the si-RIPK4 group. RIPK4, receptor-interacting protein kinase 4; OA, osteoarthritis.
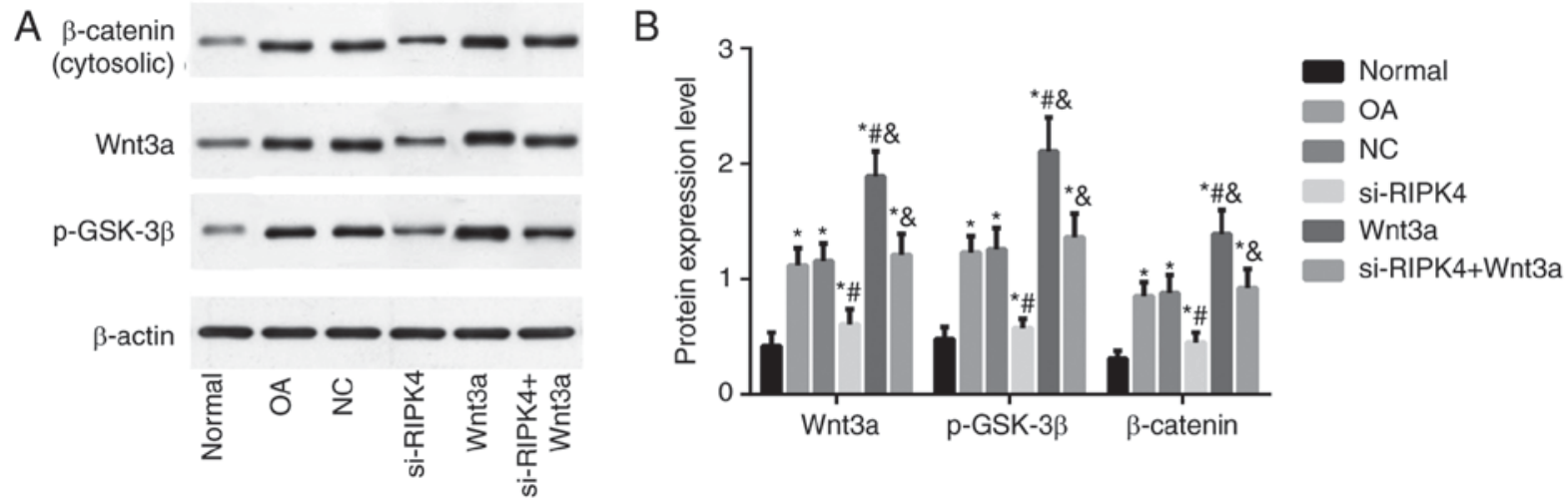

Figure 4. Expressions of Wnt/ $\beta$-catenin pathway related-proteins in each transfected group were measured by western blotting. (A) The expression of Wnt/ $\beta$-catenin pathway related-proteins detected by western blotting; (B) Gray level analysis of the expression of Wnt/ $\beta$-catenin pathway related-proteins; ${ }^{*} \mathrm{P}<0.05$ compared with Normal group; ${ }^{\prime \prime} \mathrm{P}<0.05$ compared with the OA group; ${ }^{\&} \mathrm{P}<0.05$ compared with the si-RIPK4 group. RIPK4, receptor-interacting protein kinase 4; OA, osteoarthritis.

The proliferation of chondrocytes in each transfected group was detected by MTT. Compared with the Normal group, the proliferation ability of chondrocytes in the OA group and $\mathrm{NC}$ group was obviously reduced (all $\mathrm{P}<0.05$ ), but the OA group and the NC group did not exhibit any significant difference in cell proliferation $(\mathrm{P}>0.05)$, which suggested a reduced proliferative activity of OA chondrocytes. Besides, the proliferation ability of chondrocytes was appreciably enhanced in si-RIPK4 group but obviously decreased in Wnt3a group when compared with the OA group and $\mathrm{NC}$ group $(\mathrm{P}<0.05)$. Moreover, the chondrocyte proliferation ability in the si-RIPK4+Wnt3a group was lower than in the si-RIPK4 group $(\mathrm{P}<0.05)$, indicating that the inhibition of RIPK4 expression could promote the growth of chondrocytes, and Wnt3a could reverse the effect of si-RIPK4 on chondrocyte proliferation (Fig. 2).

Chondrocytes apoptosis in each transfected group. The apoptosis of chondrocytes in each transfected group was detected by flow cytometry (Fig. 3). When compared with the Normal group, the $\mathrm{OA}$ group and $\mathrm{NC}$ group increased significantly in the rate of chondrocytes apoptosis (all $\mathrm{P}<0.05$ ), while there was no significant difference in the rate of apoptosis between the OA group and $\mathrm{NC}$ group $(\mathrm{P}>0.05)$, which indicated an elevation of the apoptosis of OA chondrocytes. Meanwhile, the lower cell apoptosis in si-RIPK4 group but higher cell apoptosis in Wnt3a group were observed as compared to OA group and $\mathrm{NC}$ group $(\mathrm{P}<0.05)$. Furthermore, the apoptosis rate of si-RIPK4+Wnt3a was significantly elevated when compared with the si-RIPK4 group, suggesting that silencing RIPK4 can effectively delay the apoptosis of chondrocytes, and Wnt3a could reverse the effect of si-RIPK4 on chondrocyte apoptosis.

Expression of Wnt/ $\beta$-catenin pathway related-proteins in each transfected group. Western blot was used to detect the expressions of $\mathrm{Wnt} / \beta$-catenin pathway related-proteins. As shown in Fig. 4, the OA group and NC group showed a remarkable increase in Wnt $3 a, \beta$-catenin, and p-GSK-3 $\beta$ proteins, as compared with the Normal group (all $\mathrm{P}<0.05$ ), but no significant difference was found in the expression of $\mathrm{Wnt} / \beta$-catenin pathway related-proteins between OA group and $\mathrm{NC}$ group (both $\mathrm{P}>0.05$ ). By comparison with OA group, the expressions of Wnt3a, $\beta$-catenin and p-GSK-3 $\beta$ were statistically lowered 


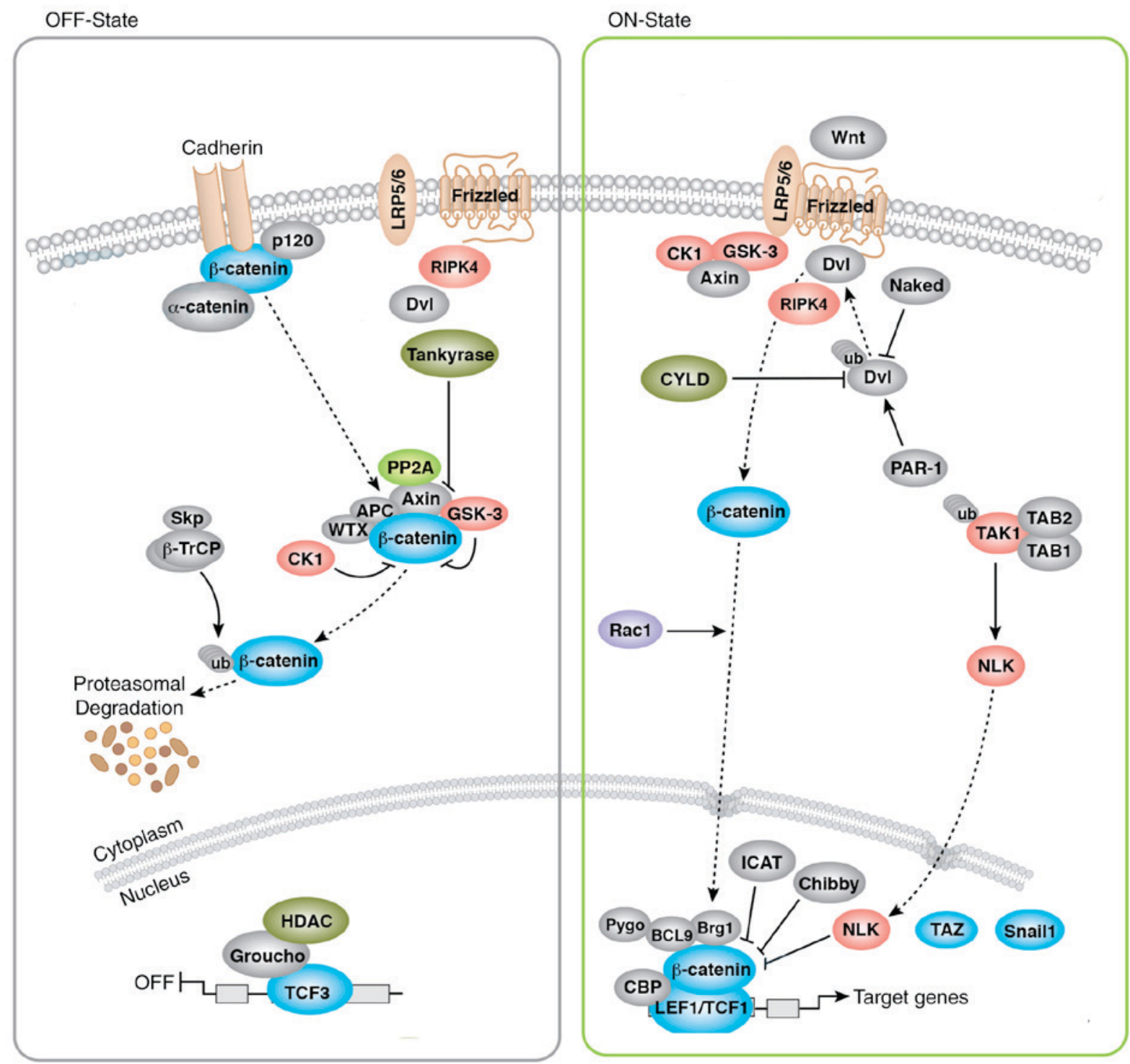

Figure 5. The model for RIPK4 function. When Wnt was stimulated, RIPK4 promoted the accumulation of cytosolic $\beta$-catenin and transcription of target genes. RIPK4, receptor-interacting protein kinase 4.

in si-RIPK4 group, but were highly upregulated in Wnt3a group. Besides, the elevated expressions of $\mathrm{Wnt} / \beta$-catenin pathway related-proteins were displayed in si-RIPK4+Wnt3a, as compared to si-RIPK4 group (all $\mathrm{P}<0.05$ ).

\section{Discussion}

$\mathrm{OA}$ is generally accepted as the most prevalent articular pathology, as well as the most frequent cause of disability, becoming a major public health concern (23). Thus, we conducted this study to better understand the molecular mechanisms implicated in the apoptosis and proliferation of chondrocytes during OA pathogenesis.

RIPK4, a key enzyme in the body, plays an important role in keratinocyte differentiation and wound healing to be involved in the process of proliferation, differentiation, and repair of the epithelium $(17,24)$. A previous study found that RIPK4 was over-expressed in oral keratinocytes, which could improve the levels of pro-inflammatory cytokines by inducing the upregulation of CCL5 and CXCL11 (25). Besides, RIPK4 was also upregulated in cervical cancer tissues and its high expression was closely related to FIGO stage and lymph node metastasis of cervical cancer (26). In addition, RIPK4 mRNA and $\beta$-catenin mRNA was reported to be remarkably higher in ovarian adenocarcinoma cells than in adjacent tissues (15). Consistent with the results of previous studies, our study also leads to the following findings that both RIPK4 mRNA and protein expressions were significantly upregulated in the articular cartilage tissues of OA patients, as well as chondrocytes, the only cellular component in cartilage, showing RIPK4 participated in the development and progression of $\mathrm{OA}$. In the meantime, by isolating chondrocytes from $\mathrm{OA}$ and normal cartilage tissues, we found that the proliferation of OA chondrocytes was decreased while the apoptotic rate was increased when compared with normal chondrocytes. During the OA development, enhanced apoptosis of chondrocytes was credited as a sign of progressive cartilage joint degeneration as indicated by Huang et al (27), and there was an explanation that OA chondrocytes still had some metabolic function although the proliferative activity of OA chondrocytes was 
relatively lower than normal ones, and, thus, a large number of intracellular proteins and RNA exhibit to be accumulated, which together with no cell division, can lead to cell necrosis or apoptosis, eventually enhancing apoptosis rate $(28,29)$. More importantly, we performed cell transfection with specific si-RNA of RIPK4 in vitro and we noticed that downregulated RIPK4 promoted the proliferation and inhibited the apoptosis of OA chondrocytes, suggesting that RIPK4 might be a potential target in $\mathrm{OA}$.

Furthermore, aberrant expression of RIPK4 has been demonstrated to have impacts on several diseases, which is a key regulatory protein of Wnt/ $\beta$-catenin signaling (16). As a classical pathway, Wnt/ $\beta$-catenin signaling is very essential for the growth, development, and death of cells $(30,31)$. It was worthy to point out that $\mathrm{Wnt} / \beta$-catenin signaling had a deep relationship with cartilage function, including the development and differentiation of cartilage $(32,33)$. In general, the key molecules in the Wnt pathway including $\beta$-catenin, GSK-3 $\beta$ and Wnt3a, in which $\beta$-catenin is the core component, essential for the upstream molecules to exert functions (34-36). There was a previous study stating that the expression of $\beta$-catenin was increased in degenerated articular chondrocytes and the loss of cartilage was linked with the activation of Wnt signaling pathways (37). As for GSK-3 $\beta$, a crucial part of the degradation complex formed by $\beta$-catenin and Axin-APC-GSK-3 $\beta$ in cytoplasm, and the inhibition of the activity of GSK-3 $\beta$ can promote the accumulation of $\beta$-catenin in cells (38-40). In our study, the protein expression of Wnt $3 a, p-\beta$-catenin and GSK-3 $\beta$ was significantly increased in OA chondrocytes, indicating the activation of the $\mathrm{Wnt} / \beta$-catenin pathway in OA. Interestingly, the activation of $\mathrm{Wnt} / \beta$-catenin signaling pathway by transfecting Wnt3a inhibited chondrocyte proliferation but promoted cell apoptosis in our study, and more importantly, Wnt3a could reverse the effect of silencing RIPK4 on chondrocyte proliferation and apoptosis. Of note, RIPK4 can bind with LRP6 to stabilize the accumulation of intracellular $\beta$-catenin, which was transferred into the nucleus to enhance classical Wnt3a pathway, and thereby promoting tumor cell growth as shown by Huang et al (15). Besides, Zhou et al reported that Tetrandrine can reduce the levels of MMPs and inflammatory factors in OA chondrocytes by inhibiting the upregulation of $\mathrm{Wnt} / \beta$-catenin pathway, and thus, exerting a protective effect on chondrocytes (41). In addition, Chen et al also reported that the over-expression of EZH2 in OA chondrocytes can elevate the H3K27me3 level on SFRP1 promoter to activate $\mathrm{Wnt} / \beta$-catenin pathway, while inhibiting EZH2 can effectively reduce the activity of $\mathrm{Wnt} / \beta$-catenin pathway and delay the progression of OA (42). Similarly, our study also observed that silencing RIPK4 appreciably reduced Wnt3a, $\beta$-catenin, and p-GSK-3 $\beta$ expressions in OA chondrocytes, suggesting that downregulating RIPK4 effectively inhibited Wnt $/ \beta$-catenin pathway and promoted the proliferation and inhibit the apoptosis of OA chondrocytes by regulating the transcription of downstream target genes related to cell proliferation and apoptosis, as demonstrated in Fig. 5. Notably, previous evidence also pointed out that specifically activation of Wnt/ $\beta$-catenin pathway in chondrocytes in adult mice resulted in the chondrocyte differentiation and the development of an OA-like phenotype via upregulation of chondrocyte marker genes, suggesting that the $\mathrm{Wnt} / \beta$-catenin pathway may serve as a potential therapeutic target in vivo (43). While RIPK4 could mediate Wnt/ $\beta$-catenin pathway in our study, thus we made the hypotheses that RIPK4 might to be a potential new target for the treatment of OA, which we need to be investigated in depth in the future studies. In summary, our study found the increased expression of RIPK4 in OA. Besides, silencing RIPK4 expression in OA chondrocytes can suppress the activation of Wnt/ $\beta$-catenin pathway, hence promoting the proliferation and inhibiting the apoptosis of chondrocytes, to exert protective roles in $\mathrm{OA}$.

\section{Acknowledgements}

The authors appreciate the reviewers for their suggestive and useful comments in this study.

\section{References}

1. Collison J: Osteoarthritis: Removing old chondrocytes to combat disease. Nat Rev Rheumatol 73: 388, 2017.

2. Johnson VL and Hunter DJ: The epidemiology of osteoarthritis. Best Pract Res Clin Rheumatol 28: 5-15, 2014.

3. Lawrence RC, Felson DT, Helmick CG, Arnold LM, Choi H, Deyo RA, Gabriel S, Hirsch R, Hochberg MC, Hunder GG, et al: Estimates of the prevalence of arthritis and other rheumatic conditions in the United States. Part II. Arthritis Rheum 58: 26-35, 2008.

4. Gomes-Neto M, Araujo AD, Junqueira ID, Oliveira D, Brasileiro A and Arcanjo FL: Comparative study of functional capacity and quality of life among obese and non-obese elderly people with knee osteoarthritis. Rev Bras Reumatol Engl Ed 56: 126-130, 2016 (In English, Portuguese).

5. Iannone F and Lapadula G: The pathophysiology of osteoarthritis. Aging Clin Exp Res 15: 364-372, 2003.

6. Neogi T and Zhang Y: Epidemiology of osteoarthritis. Rheum Dis Clin North Am 39: 1-19, 2013.

7. Michael JW, Schluter-Brust KU and Eysel P: The epidemiology, etiology, diagnosis, and treatment of osteoarthritis of the knee. Dtsch Arztebl Int 107: 152-162, 2010.

8. Alcaraz MJ, Megias J, Garcia-Arnandis I, Clérigues V and Guillén MI: New molecular targets for the treatment of osteoarthritis. Biochem Pharmacol 80: 13-21, 2010.

9. Zhang D, Lin J and Han J: Receptor-interacting protein (RIP) kinase family. Cell Mol Immunol 7: 243-249, 2010.

10. Bhr C, Rohwer A, Stempka L, Rincke G, Marks F and Gschwendt M: DIK, a novel protein kinase that interacts with protein kinase Cdelta. Cloning, characterization, and gene analysis. J Biol Chem 275: 36350-36357, 2000.

11. Chen L, Haider K, Ponda M, Cariappa A, Rowitch D and Pillai S: Protein kinase C-associated kinase (PKK), a novel membrane-associated, ankyrin repeat-containing protein kinase. J Biol Chem 276: 21737-21744, 2001.

12. Meylan E, Martinon F, Thome M, Gschwendt M and Tschopp J: RIP4 (DIK/PKK), a novel member of the RIP kinase family, activates NF-kappa $\mathrm{B}$ and is processed during apoptosis. EMBO Rep 3: 1201-1208, 2002.

13. Meylan E and Tschopp J: The RIP kinases: Crucial integrators of cellular stress. Trends Biochem Sci 30: 151-159, 2005.

14. Adams S and Munz B: RIP4 is a target of multiple signal transduction pathways in keratinocytes: Implications for epidermal differentiation and cutaneous wound repair. Exp Cell Res 316: 126-137, 2010.

15. Huang X, McGann JC, Liu BY, Hannoush RN, Lill JR, Pham V, Newton K, Kakunda M, Liu J, Yu C, et al: Phosphorylation of Dishevelled by protein kinase RIPK4 regulates Wnt signaling. Science 339: 1441-1445, 2013.

16. Liu DQ, Li FF, Zhang JB, Zhou TJ, Xue WQ, Zheng XH, Chen YB, Liao XY, Zhang L, Zhang SD, et al: Increased RIPK4 expression is associated with progression and poor prognosis in cervical squamous cell carcinoma patients. Sci Rep 5: 11955, 2015.

17. Rountree RB, Willis CR, Dinh H, Blumberg H, Bailey K, Dean C Jr, Peschon JJ and Holland PM: RIP4 regulates epidermal differentiation and cutaneous inflammation. J Invest Dermatol 130: 102-112, 2010. 
18. Adams S, Pankow S, Werner S and Munz B: Regulation of NF-kappaB activity and keratinocyte differentiation by the RIP4 protein: Implications for cutaneous wound repair. J Invest Dermatol 127: 538-544, 2007.

19. Rose J, Söder S, Skhirtladze C, Schmitz N, Gebhard PM, Sesselmann S and Aigner T: DNA damage, discoordinated gene expression and cellular senescence in osteoarthritic chondrocytes. Osteoarthritis Cartilage 20: 1020-1028, 2012.

20. The helsinki declaration of the world medical association (WMA). Ethical principles of medical research involving human subjects. Pol Merkur Lekarski 36: 298-301, 2014 (In Polish)

21. Dias RC, Dias JM and Ramos LR: Impact of an exercise and walking protocol on quality of life for elderly people with OA of the knee. Physiother Res Int 8: 121-130, 2003.

22. Tuo YL, Li XM and Luo J: Long noncoding RNA UCA1 modulates breast cancer cell growth and apoptosis through decreasing tumor suppressive miR-143. Eur Rev Med Pharmacol Sci 19: 3403-3411, 2015.

23. Ebrahimzadeh MH, Makhmalbaf H, Birjandinejad A and Soltani-Moghaddas SH: Cross-cultural adaptation and validation of the persian version of the oxford knee score in patients with knee osteoarthritis. Iran J Med Sci 39: 529-535, 2014.

24. Holland P, Willis C, Kanaly S, Glaccum M, Warren A, Charrier K, Murison J, Derry J, Virca G, Bird T and Peschon J: RIP4 is an ankyrin repeat-containing kinase essential for keratinocyte differentiation. Curr Biol 12: 1424-1428, 2002.

25. Kwa MQ, Scholz GM and Reynolds EC: RIPK4 activates an IRF6-mediated proinflammatory cytokine response in keratinocytes. Cytokine 83: 19-26, 2016.

26. Azizmohammadi S, Azizmohammadi S, Safari A, Kaghazian M, Sadrkhanlo M, Behnod V and Seifoleslami M: High-level expression of RIPK4 and EZH2 contributes to lymph node metastasis and predicts favorable prognosis in patients with cervical cancer. Oncol Res 25: 495-501, 2017

27. Huang Z, Li J, Du S, Chen G, Qi Y, Huang L, Xiao L and Tong P: Effects of UCP4 on the proliferation and apoptosis of chondrocytes: Its possible involvement and regulation in osteoarthritis PLoS One 11: e0150684, 2016.

28. Schroeppel JP, Crist JD, Anderson HC and Wang J: Molecular regulation of articular chondrocyte function and its significance in osteoarthritis. Histol Histopathol 26: 377-394, 2011

29. Zhong JH, Li J, Liu CF, Liu N, Bian RX, Zhao SM, Yan SY and Zhang YB: Effects of microRNA-146a on the proliferation and apoptosis of human osteoarthritis chondrocytes by targeting TRAF6 through the NF- $\mathrm{KB}$ signalling pathway. Biosci Rep 37 : pii: BSR20160578, 2017.

30. Wang Y, Li YP, Paulson C, Shao JZ, Zhang X, Wu M and Chen W: Wnt and the Wnt signaling pathway in bone development and disease. Front Biosci (Landmark Ed) 19: 379-407, 2014

31. Lad EM, Cheshier SH and Kalani MY: Wnt-signaling in retinal development and disease. Stem Cells Dev 18: 7-16, 2009.
32. Corr M: Wnt-beta-catenin signaling in the pathogenesis of osteoarthritis. Nat Clin Pract Rheumatol 4: 550-556, 2008.

33. Johnson ML and Kamel MA: The Wnt signaling pathway and bone metabolism. Curr Opin Rheumatol 19: 376-382, 2007.

34. Reinhold MI, Kapadia RM, Liao Z and Naski MC: The Wnt-inducible transcription factor Twistl inhibits chondrogenesis. J Biol Chem 281: 1381-1388, 2006.

35. Nalesso G, Sherwood J, Bertrand J, Pap T, Ramachandran M, De Bari C, Pitzalis C and Dell'accio F: WNT-3A modulates articular chondrocyte phenotype by activating both canonical and noncanonical pathways. J Cell Biol 193: 551-564, 2011.

36. Funck-Brentano T, Bouaziz W, Marty C, Geoffroy V, Hay E and Cohen-Solal M: Dkk-1-mediated inhibition of Wnt signaling in bone ameliorates osteoarthritis in mice. Arthritis Rheumatol 66: 3028-3039, 2014

37. Rockel JS, Yu C, Whetstone H, Craft AM, Reilly K, Ma H, Tsushima H, Puviindran V, Al-Jazrawe M, Keller GM and Alman BA: Hedgehog inhibits $\beta$-catenin activity in synovial joint development and osteoarthritis. J Clin Invest 126: 1649-1663, 2016.

38. Delgado E, Bahal R, Yang J, Lee JM, Ly DH and Monga SP: $\beta$-Catenin knockdown in liver tumor cells by a cell permeable gamma guanidine-based peptide nucleic acid. Curr Cancer Drug Targets 13: 867-878, 2013.

39. Gosens R, Meurs $H$ and Schmidt M: The GSK-3/beta-catenin-signalling axis in smooth muscle and its relationship with remodelling. Naunyn Schmiedebergs Arch Pharmacol 378: 185-191, 2008.

40. Cui XP, Xing Y, Chen JM, Dong SW, Ying DJ and Yew DT: Wnt/beta-catenin is involved in the proliferation of hippocampal neural stem cells induced by hypoxia. Ir J Med Sci 180: 387-393, 2011.

41. Zhou X, Li W, Jiang L, Bao J, Tao L, Li J and Wu L: Tetrandrine Inhibits the Wnt/ $\beta$-catenin signalling pathway and alleviates osteoarthritis: An in vitro and in vivo study. Evid Based Complement Alternat Med 2013: 809579, 2013.

42. Chen L, Wu Y, Wu Y, Wang Y, Sun L and Li F: The inhibition of EZH2 ameliorates osteoarthritis development through the Wnt/ß-catenin pathway. Sci Rep 6: 29176, 2016.

43. Zhu M, Tang D, Wu Q, Hao S, Chen M, Xie C, Rosier RN, O'Keefe RJ, Zuscik M and Chen D: Activation of beta-catenin signaling in articular chondrocytes leads to osteoarthritis-like phenotype in adult beta-catenin conditional activation mice. J Bone Miner Res 24: 12-21, 2009.

(i) $\Theta$ This work is licensed under a Creative Commons Attribution-NonCommercial-NoDerivatives 4.0 International (CC BY-NC-ND 4.0) License. 